\title{
Enhancing the Capability of a Ground-Based Optical Telescope for Thai National Space objects Observation ${ }^{\dagger}$
}

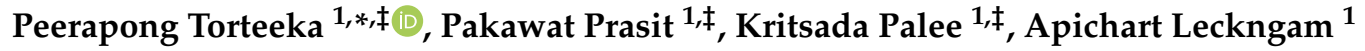 \\ and Patcharin Kamsing ${ }^{2}$ \\ 1 National Astronomical Research Institute of Thailand (NARIT), Chiang-Mai 50180, Thailand; \\ pakawat@narit.or.th (P.P.); kritsada.p@narit.or.th (K.P.); apichat@narit.or.th (A.L.) \\ 2 King Mongkut's Institute of Technology Ladkrabang (KMITL), Bangkok 10520 Thailand; \\ patcharin.ka@kmitl.ac.th \\ * Correspondence: peerapong@narit.or.th; Tel.: +66-053-225-569 \\ + Presented at the Innovation Aviation \& Aerospace Industry-International Conference 2020 (IAAI 2020), \\ Chumphon, Thailand, 13-17 January 2020. \\ $\ddagger$ These authors contributed equally to this work.
}

Published: 7 January 2020

\begin{abstract}
Nowadays, the space operations environment have to face with space safety problems because of the growing of space debris in resident of space objects (RSOs) that can cause a catastrophic collision. In order to prevent debris-related risks in operational orbit, ground-based passive optical telescope network were used as a primary equipment for space debris observation due to the lowest maintenance costs. Furthermore, in technical, a precise tracking (position and velocity) of space objects can be beneficial towards not only orbit determination but also estimation spacecraft collision probability especially, in Low-Earth Orbit regime. National Astronomical Research Institute of Thailand (NARIT) has long experience operate in an observatory to perform both passive \& active optical instruments for astrophysics and space sciences missions. In this research, based on Thai National Space objects Observation (TNSO) project, we re-establish the basic understanding of satellite tracking, optical subsystem integration and demonstration a framework so as to enhance the capability of telescope servo control subsystem. We describe the specific solutions adopted for continuous tracking mode and the results obtained during the commissioning of an alt-azimuth mounting equipped with 0.7 meter optical aperture telescope. The observation system can be performed with negligible as continuous tracking error. This contribution will present some of the experimental results and plans for further measurement campaigns.
\end{abstract}

Keywords: space debris; passive optical telescope; space situation awareness

\section{Introduction}

According to the index of Union of Concerned Scientists (UCS), there are 4987 objects currently in orbit around Earth however, only 1957 are operational satellites. Moreover, the unexpected on-orbit spacecraft collision (Cosmos-2251 and Iridium-33) and the testing of anti-satellite missile have caused an immense number of fragmentation debris in RSOs that are the part which generates Kessler syndrome [1]. In the new space era, the private sector of space industry are growing by leaps and bounds. They have been proposed lunching over 42,000 constellation satellites in orbit for this decade especially, the SpaceX V-band \& Starlink project [2]. By following this, the U.S. Air Force tracked a priority item of space objects and categorized them against known space debris. The orbital parameters of Earth-orbiting objects have primarily been provided by the North American Aerospace Defense Command (NORAD); however, space target tracking with known orbital parameters can be achieved relatively easily. 


\section{Methodology}

Enhancing the capability of tracking procedure is necessary for TNSO telescope as one of space situation awareness system (SSA). In detail, the mechanical structure of alt-azimuth mounting telescope can rotate in the azimuth and elevation axes via a simple position controller. We found that, based on continuous tracking mode as illustrated in Figure 1, although the accuracy of the Two-Lines Element (TLE) data is generally good enough to detect the space target in the Field-of-View (FoV) through the Simplified General Perturbations (SGP4) orbital model prediction [3], the space target is often not very well centered in the image and may even slowly drift out of the FoV during the tracking procedure.

In the continuous tracking mode, most single space target observed from the time of observation initiation tend to appear in the central image FoV scanned region. To evaluate a close-loop tracking architecture [4], a recognizing of space objects with known orbital parameters is prioritized. Initially, the telescope guidance system calculates the approximate candidate space target both position and velocity, while the two perpendicular axes of the telescope slew to the expectation point, where the target is expected to cross the image FoV within a few moments. When the object crosses the FoV, the camera is operated with a short exposure time to capture the space object following the SGP4 orbital trajectory through the kinematic of telescope. Therefore, this paper investigates a overall system architecture, the proposed strategy emphasizes real-time tracking. The primary challenges hindering this development are difficulties regarding unstable velocity of moving object and the need for more robust tracking.

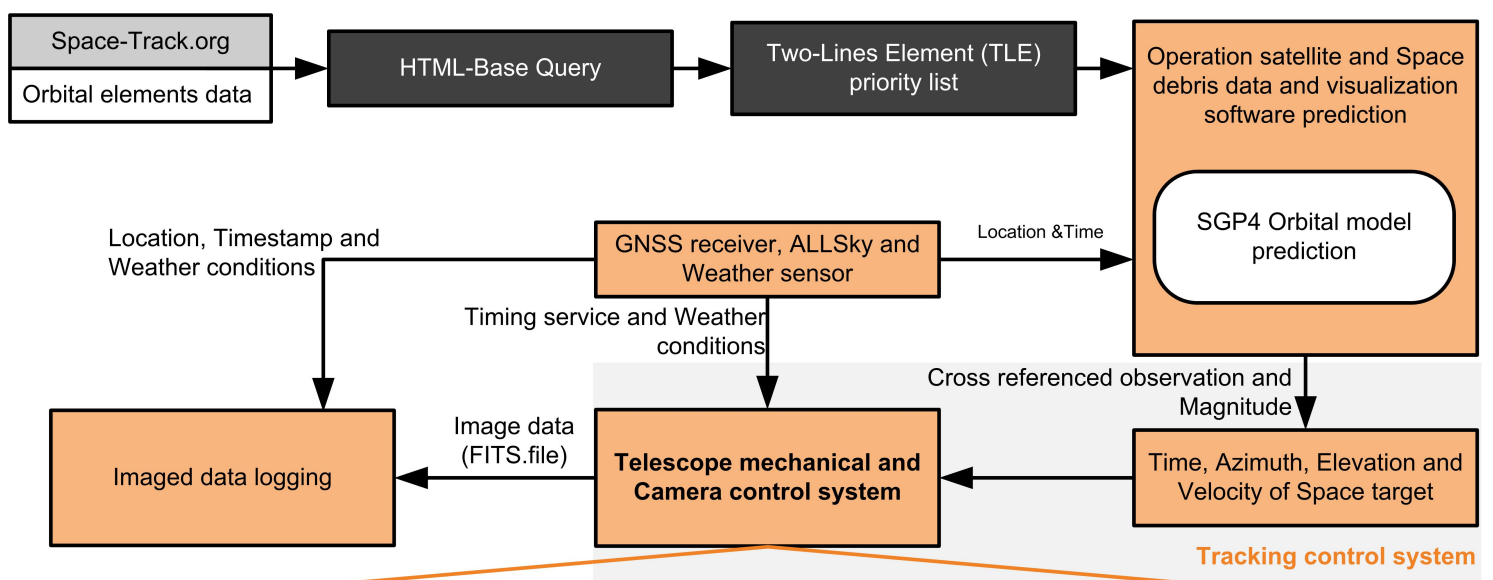

+ Dome rotation control system + Close-loop space target tracking system + Graphic User Interface + Camera control system

Figure 1. System architecture of Thai National Space objects Observation (TNSO) telescope.

\section{Experimental Results}

In our experiment, alt-azimuth TNSO telescope has been installed at Princess Sirindhorn AstroPark observatory, it was located close to the equator as the northern area of Thailand (WGS84) at Lat. 18.8531; Long. 98.9583 degrees and alt. 347 meter. The TNSO telescope used the Thai Robotic Telescope (TRT) as the high-level software operation [5] that consist of astrometry, communication system, data handling, time system, pointing model analysis and weather monitoring etc. In technical detail, TNSO telescope equipped with a complementary metal-oxide semiconductor (CMOS)-type high-resolution sensor that stored as Flexible Image Transport System (FITS)-type gray-scale images with range [0, 255] and the Audio Video Interleaved (AVI) format for video data. The $0.7 \mathrm{~m}$ TNSO telescope specifications are listed in Table 1, this observatory mainly used for precise tracking and improving the accuracy to target data. 
Table 1. Specifications of TNSO telescope .

\begin{tabular}{lll}
\hline Item & Function & Parameters \\
\hline Tube & Optical system & Aperture size: $0.7 \mathrm{~m}$, refractor. \\
& & Focal length: $4540 \mathrm{~mm}$. \\
& Image size radii: $\approx 5.85 \mu \mathrm{m}$. \\
\hline CMOS-type & Image sensor & Resolution: $1936 \times 1216$ pixels \\
& & image FoV: $0.1431^{\circ} \times 0.0899^{\circ}$ \\
\hline Mount & Support part & Track speed: $\geq 5^{\circ} \mathrm{s}^{-1}$ \\
& & Track acceleration: $2^{\circ} \mathrm{s}^{-2}$ \\
& & Position accuracy: 0.5 arc-sec. \\
& Elevation range: $13^{\circ}-88^{\circ}$ \\
& & Azimuth range: $0^{\circ}- \pm 270^{\circ}$ \\
\hline Software & Observation control & Software: Thai Robotic Telescope (TRT) \\
& & Input data: Location, time and NORAD-TLE data. \\
& & Positioning: Astronomical/axial. \\
& & Database: USNO stars catalog. \\
\hline
\end{tabular}

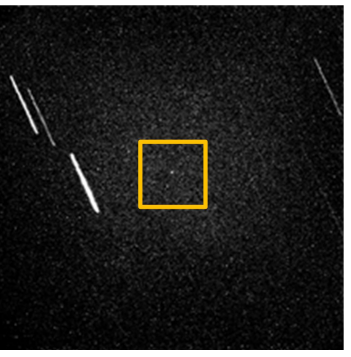

(a) NORAD\#23628

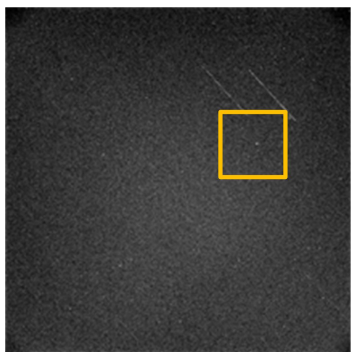

(b) NORAD\#43706

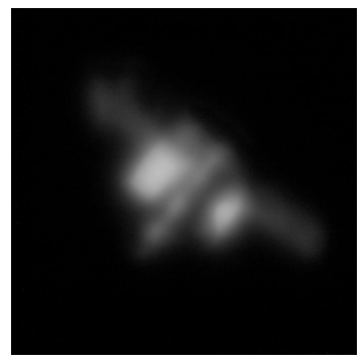

(e) NORAD\#25544

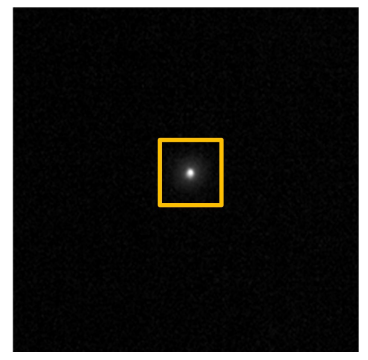

(c) NORAD\#1361

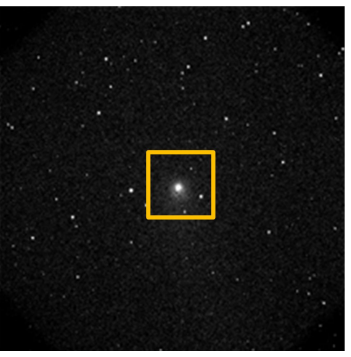

(f) NEO

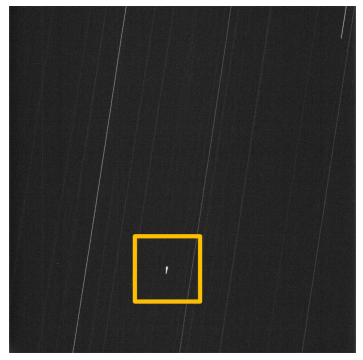

(d) NORAD\#40020

Figure 2. Tracking datasets of space targets with known orbital parameters labeled by $(\mathbf{a}, \mathbf{b})$ are Geostationary and Medium Earth orbit objects respectively. (c,d) are Low Earth orbit object. Lastly, (e) is the Near-Earth Object (NEO): Africano. Orange rectangles: Observations with Regions of Interest (ROIs) around space targets. Observation date: July-September 2019.

The design also take into the survey ability so as to guiding and finding the space objects with $0.15 \mathrm{~m}, \mathrm{f} / 8$ reflector telescope equipped with monochrome CMOS sensor by $1.2 \times 10^{6}$ pixels as a piggyback installation. In the resulting of sequential images as illustrated in Figure 2, the extended target have similar appearances in terms of the point-spread function. In the ideal case, the degree of spreading (blurring) and shape of point-like objects is a measure of the control system behavior which, basically. 


\section{Conclusions}

In this article, we primary presents the progress of TNSO project, an objective for the development of modern ground-based space surveillance networks in order to support Thai national space policy. For the first demonstration, the space target images were accurately tracked through the proposed software and hardware architectures via ground-based optical observation located at Chiang-Mai Thailand. Technically, in space objects observation, the optical system require an imaging sensor with fast processing and readout within an epochs of the exposures time, especially the space object in Low Earth orbit regime. Therefore, in this application, the CMOS sensor can be appropriately performed with electronic shutters and flexible readout capabilities. However, the tracking performance of this equipment primarily depends on size and brightness of space objects, which appears on screen. In the real-time observation procedure based on stacking method in short exposure time, the space target and stellar background will similarly appear in point-spread function with difference levels of Signal-to-Noise Ratio under the conditions of background interference, which is difficult to recognize. Therefore, future guideline development of this project should spend more software integration techniques based on computer vision and parameter estimation.

Author Contributions: P.T. conceived and designed the telescope control system strategy; P.P. performed the high-level TRT software management; K.P. designed Software's Graphic User Interface and signal processing. P.T., P.P. and K.P. analyzed the data; A.L. and P.K. contributed materials and analysis tools; and P.T. wrote the paper.

Acknowledgments: The authors would like to extend their gratitude to Sittiporn Channumsin and Suwat Sreesawet of the GISTDA Academy, Geo-Informatics and Space Technology Development Agency (GISTDA), Siracha- Thailand, for their assistance with the reference data and space policy. The authors would also like to thank to Manop Aorpimai, Director-General of Strategic Planning and Program Management Department, Asia-Pacific Space Cooperation organization (APSCO), Beijing- China, and anonymous reviewers for their insightful comments, which have significantly improved this paper.

\section{References}

1. Kessler, D.J.; Johnson, N.L.; Liou, J.; Matney, M. The kessler syndrome: implications to future space operations. Adv. Astronaut. Sci. 2010, 137, 2010.

2. Muelhaupt, T.J.; Sorge, M.E.; Morin, J.; Wilson, R.S. Space traffic management in the new space era. J. Space Saf. Eng. 2019, 6, 80-87.

3. Vallado, D.; Crawford, P.; Hujsak, R.; Kelso, T. Revisiting spacetrack report\# 3. In Proceedings of the AIAA/AAS Astrodynamics Specialist Conference and Exhibit, Keystone, CO, USA, 21-24 August 2006; p. 6753.

4. Thinh, N.H.Q.; Choi, J.G.; Kim, W.H. Design and Implementation of FFPIV Scheme for Closed Loop Motion Controller. Intell. Control. Autom. 2014, 5, 35.

5. Lam, M.C.; Vijarnwannaluk, B.; Butpan, P.; Copperwheat, C.M.; Piascik, A.S.; Sawangwit, U.; Smith, R.J.; Steele, I.A. Laying the groundwork for the development of the data archive of the new robotic telescope. In Software and Cyberinfrastructure for Astronomy V; International Society for Optics and Photonics: Bellingham, WA, UDA, 2018; Volume 10707, p. 1070721.

(C) 2020 by the authors. Licensee MDPI, Basel, Switzerland. This article is an open access article distributed under the terms and conditions of the Creative Commons Attribution (CC BY) license (http:// creativecommons.org/licenses/by/4.0/). 